\title{
THE ZERO, POLE AND ORDER OF MEROMORPHIC SOLUTIONS OF DIFFERENTIAL EQUATIONS WITH MEROMORPHIC COEFFICIENTS*
}

\author{
ZONG-XUAN CHEN
}

\begin{abstract}
In this paper, we investigate the complex oscillation of non-homogeneous linear differential equations with meromorphic coefficients under substracting the condition that all solutions of differential equation are meromorphic functions.
\end{abstract}

\section{Introduction and results}

Consider non-homogeneous linear differential equations of the form

$$
f^{(k)}+b_{k-1} f^{(k-1)}+\cdots+b_{0} f=H(z) \quad(k \geqq 1)
$$

where $b_{k-\jmath}(j=1, \cdots, k)$ are rational functions, $H(z)$ is a meromorphic function. Z.-X. Chen and S.-A. Gao proved in [3].

THEOREM A. Let $b_{k-},(j=1, \cdots, k)$ be rational functions having a pole at $\infty$ of order $n_{k-\jmath} \geqq 0, k \geqq 1, H(z)$ be a meromorphic function, $\sigma(H)=\beta$ satisfying

$$
1+\max _{1 \leqq j \leq k} n_{k-j} / j<\beta<\infty .
$$

If all solutions $f$ of the differential equation (1.1) are meromorphic functions, then

(a) $\sigma(f)=\beta$.

(b) $\lambda(1 / f)=\lambda(1 / H), \tilde{\lambda}(1 / f)=\tilde{\lambda}(1 / H)$. If $\lambda(H)>\lambda(1 / H)$, then $\lambda(f) \geqq \lambda(H)$.

(c) If $\beta>\max \{\lambda(H), \lambda(1 / H)\}$, then all solutions of (1.1) satisfy $\hat{\lambda}(f)=\lambda(f)=$ $\sigma(f)=\beta$, except at most a possible one. The possible exceptional one $f_{0}$ satisfies $\lambda\left(f_{0}\right)<\beta$.

* Project supported by the National Natural Science Foundation of China.

Keywords: non-homogeneous linear differential equation, meromorphic function, zero, pole, order.

AMS No.: 34A20, 30D35.

Received June 9, 1995; revised April, 19, 1996. 
THEOREM B. Let $b_{k-\jmath}(j=1, \cdots, k)$ be rational functions having a pole at $\infty$ of order $n_{k-\jmath} \geqq 0, k \geqq 1, H(z) \neq 0$ be a meromorphic function satısfying $\sigma(H)=$ $\beta \leqq 1+\max _{1 \leqq j \leqq k} n_{k-j} / j$. If all solutions $f$ of (1.1) are meromorphic functions, then

(a) $\beta \leqq \sigma(f) \leqq 1+\max _{1 \leqq j \leqq k} n_{k-j} / j$.

(b) $\lambda(1 / f)=\lambda(1 / H), \bar{\lambda}(1 / f)=\bar{\lambda}(1 / H)$. If $\lambda(H)>\lambda(1 / H)$ then $\lambda(f) \geqq \lambda(H)$.

(c) If $\sigma(f)>\beta$, then $\bar{\lambda}(f)=\lambda(f)=\sigma(f)$.

In this paper, we use the same notations as in $[1]$, i.e. $\lambda(f)$ and $\bar{\lambda}(f)$ to denote respectively the exponents of convergence of the zero-sequence and the sequence of distinct zeros of $f(z), \lambda(1 / f)$ and $\bar{\lambda}(1 / f)$ to denote respectively the exponents of convergence of the pole-sequence and the sequence of distinct poles of a meromorphic function $f(z), \sigma(f)$ to denote the order of growth of $f(z)$. And we use the standard notations of the Nevanlinna theory (e.g. see [5]).

By a fundamental theory of the differential equation with complex coefficients, we know that all solutions of linear differential equation with entire coefficients are entire functions. But a solution of linear differential equation with meromorphic coefficients is not always a meromorphic function. For example, $f_{1}=\exp \{1 / z\}+e^{z}$ and $f_{2}=e^{z}$ are all solutions of the equation

$$
f^{\prime \prime}+\left(z^{3}+z^{2}\right) f^{\prime}+\left(z+1-\frac{1}{z^{4}}-\frac{2}{z^{3}}\right) f=\left(z^{3}+z^{2}+z+2-\frac{1}{z^{4}}-\frac{2}{z^{3}}\right) e^{z}
$$

but $f_{1}$ is not a meromorphic function. Therefore in Theorems $\mathrm{A}$ and $\mathrm{B}$, the condition that all solutions of (1.1) are meromorphic functions is very rigorous. In this paper, we will substract this condition in Theorems A and B to generalize Theorems $\mathrm{A}$ and $\mathrm{B}$.

We will prove the following theorems.

THEOREM 1. Suppose that $b_{k-\jmath}(j=1, \cdots, k)$ are rational functions having a pole at $\infty$ of order $n_{k-\jmath} \geqq 0, k \geqq 1, H(z)$ is a meromorphic function, $\sigma(H)=\beta$ satisfying (1.2). If (1.1) has a meromorphic solution $f$, then

(a) $\sigma(f)=\beta$.

(b) $\lambda(1 / f)=\lambda(1 / H), \bar{\lambda}(1 / f)=\bar{\lambda}(1 / H)$. If $\lambda(H)>\lambda(1 / H)$, then $\lambda(f) \geqq \lambda(H)$.

(c) If $\beta>\max \{\lambda(H), \lambda(1 / H)\}$, then all meromorphic solutions of (1.1) satisfy $\bar{\lambda}(f)=\lambda(f)=\sigma(f)=\beta$, except at most one $f_{0}$ satisfying $\lambda\left(f_{0}\right)<\beta$.

THEOREM 2. Suppose that $b_{k-\jmath}(\jmath=1, \cdots, k)$ are rational functions having a pole at $\infty$ of order $n_{k-\jmath} \geqq 0, k \geqq 1, H(z) \neq 0$ is a meromorphic function satisfying $\sigma(H)=\beta \leqq 1+\max _{1 \leq \jmath \leq k} n_{k-j} / \jmath$. If (1.1) has a meromorphic solution $f$, then

(a) $\beta \leqq \sigma(f) \leqq 1+\max _{1 \leqq \jmath \leqq k} n_{k-j} / j$.

(b) $\lambda(1 / f)=\lambda(1 / H), \bar{\lambda}(1 / f)=\bar{\lambda}(1 / H)$. If $\lambda(H)>\lambda(1 / H)$, then $\lambda(f) \geqq \lambda(H)$.

(c) If $\boldsymbol{\sigma}(f)>\beta$, then $\bar{\lambda}(f)=\lambda(f)=\boldsymbol{\sigma}(f)$.

Example having an exceptional solution in Theorem 1(c). 
The equation

$$
f^{\prime \prime}+f^{\prime}-2(z+1) f=\left(\frac{1+\sin ^{2} z}{\cos ^{3} z}+\frac{(4 z+1) \sin z}{\cos ^{2} z}+\frac{4 z^{2}}{\cos z}\right) e^{z^{2}}
$$

satisfies the additional hypothesis of Theorem 1 (c). And the equation has exceptional solution $f_{0}=(1 / \cos z) e^{z^{2}}$, where $\sigma\left(f_{0}\right)=2, \lambda\left(1 / f_{0}\right)=1, \lambda\left(f_{0}\right)=0<\sigma\left(f_{0}\right)$.

\section{Lemmas and preliminaries}

THEOREM C (Borel, see Theorem 5.13 in [7] or 2.6.18. Lemma in [2, P. 21]). Suppose that $Q(z)$ is canonical product formed with $\left\{z_{n} ; n=1,2, \cdots\right\}\left(z_{n} \neq 0\right)$ and $\lambda(Q)=\beta<\infty$. Set $O_{n}=\left\{z:\left|z-z_{n}\right|<\left|z_{n}\right|^{-\alpha}\right\} .(\alpha(>\beta)$ is a constant $)$ then for any given $\varepsilon>0$,

holds for $z \notin \bigcup_{n=1}^{\infty} O_{n}$.

$$
|Q(z)| \geqq \exp \left\{-|z|^{\beta+\varepsilon}\right\}
$$

Theorem D (See [6, P. 19] or 2.3.6* in [2, P. 13]). Suppose that $w(z)$ is a finite order entire function, $\mu(r)$ is the maximum term of the power series of $w(z)$, then

$$
\lim _{r \rightarrow \infty} \log M(r, w) / \log \mu(r)=1 \text {. }
$$

LEMMA 1. Suppose that $H(z)$ is a meromorphic function, $\sigma(H)=\beta<\infty$, then for any given $\varepsilon>0$, there is a set $E_{1} \subset(1, \infty)$ that has finite linear measure and finite logarithmic measure, such that

$$
|H(z)| \leqq \exp \left\{r^{\beta+\varepsilon}\right\}
$$

holds for $|z|=r \notin[0,1] \cup E_{1}, r \rightarrow \infty$.

Proof. If $H$ has only finitely many poles, then Lemma 1 holds obviously. Now assume that $H(z)$ has infinitely many poles. Set $H(z)=h(z) /\left[z^{k} \cdot Q(z)\right]$, where $k_{1}$ is nonnegative integer, $h(z)$ is an entire function, $Q(z)$ is the canonical product formed with the nonzero poles $\left\{z_{\jmath}: j=1,2, \cdots ;\left|z_{j}\right|=r_{\jmath}, 0<r_{1} \leqq r_{2} \leqq \cdots\right\}$ of $H(z)$, hence $\sigma(h) \leqq \sigma(H)=\beta, \sigma(Q)=\lambda(Q) \leqq \beta$.

For any given $\varepsilon>0$, set $O,=\left\{z:\left|z-z_{j}\right| \leqq r_{j}^{-(\beta+\varepsilon / 2)}\right\}(j=1,2, \cdots\}$ and $O=$ $\bigcup_{j=1}^{\infty} O_{\jmath}$. Set

$$
E_{1}=\bigcup_{j=1}^{\infty}\left(r_{j}-r_{j}^{-(\beta+\varepsilon / 2)}, r_{j}+r_{j}^{-(\beta+\varepsilon / 2)}\right)
$$

Since

$$
\sum_{j=1}^{\infty} 1 / r_{j}^{\beta+\varepsilon / 2}=d<\infty
$$

we know that linear measure of $E_{1}, m E_{1}=2 d<\infty$. For $|z|=r \notin E_{1} \cup[0,1]$, we have from Theorem $\mathrm{C}$

$$
|Q(z)| \geqq \exp \left\{-r^{\beta+\varepsilon / 2}\right\}
$$


Hence

$$
|H(z)| \leqq \exp \left\{2 r^{\beta+\varepsilon / 2}\right\} / r^{k} \leqq \exp \left\{r^{\beta+\varepsilon}\right\}
$$

holds for $|z|=r \notin E_{1} \cup[0,1], r \rightarrow \infty$.

Now we prove the logarithmic measure of $E_{1}, \operatorname{lm} E_{1}<\infty$. From

$$
\begin{aligned}
\operatorname{lm} E_{1} & =\sum_{j=1}^{\infty}\left[\log \left(r_{j}+r_{j}^{-(\beta+\varepsilon / 2)}\right)-\log \left(r_{j}-r_{j}^{-(\beta+\varepsilon / 2)}\right)\right] \\
& =\sum_{j=1}^{\infty} \log \left(1+\frac{2 r_{j}^{-(\beta+\varepsilon / 2)}}{r_{j}-r_{j}^{-(\beta+\varepsilon / 2)}}\right),
\end{aligned}
$$

and for sufficiently large $r_{0}$

$$
\log \left(1+\frac{2 r_{j}^{-(\beta+\varepsilon / 2)}}{r_{j}-r_{j}^{-(\beta+\varepsilon / 2)}}\right) \leqq \frac{2 r_{j}^{-(\beta+\varepsilon / 2)}}{r_{j}-r_{j}^{-(\beta+\varepsilon / 2)}} \leqq 2 r_{j}^{-(\beta+\varepsilon / 2)}
$$

we have $\operatorname{lm} E_{1}<\infty$ by (2.1).

LEMMA 2. Suppose that $g(z)$ is a transcendental entire function, $\sigma(g)=\alpha<\infty$, then there is a set $E_{2} \subset(1, \infty)$ that has infinite logarithmic measure such that

$$
\lim _{\substack{r \rightarrow \infty \\ r \in E_{2}}} \frac{\log \log M(r, g)}{\log r}=\lim _{\substack{r \rightarrow \infty \\ r \in E_{2}}} \frac{\log \nu_{g}(r)}{\log r}=\alpha
$$

where $\nu_{g}(r)$ denotes the centralindex of $g(z)$.

Proof. By $\sigma(g)=\alpha$, there exists $\left\{r_{n}\right\} \quad\left(r_{n} \rightarrow \infty\right)$, such that

$$
\lim _{r_{n} \rightarrow \infty} \frac{\log \log M\left(r_{n}, g\right)}{\log r_{n}}=\alpha .
$$

Setting $E_{2} \subset(1,+\infty), E_{2}$ has the following properties: (a) If the sequence $\left\{r_{n}\right\}$ satisfies (2.2), then $\left\{r_{n}\right\} \subset E_{2}$. (b) If a sequence $\left\{r_{n}\right\} \subset E_{2}\left(r_{n} \rightarrow \infty\right)$, then (2.2) holds for $\left\{r_{n}\right\}$. Now we affirm that logarithmic measure of $E_{2}, \operatorname{lm} E_{2}=\infty$. In fact, if $\operatorname{lm} E_{2}=\delta<\infty$, then from the definition of $E_{2}$, we have

$$
\varlimsup_{\substack{r \rightarrow \infty \\ r \in(1, \infty)-E_{2}}} \frac{\log \log M(r, g)}{\log r}=\alpha_{1}<\alpha .
$$

Now for a given $\left\{r_{n}^{\prime}\right\} \subset(1, \infty), r_{n}^{\prime} \rightarrow \infty$, there exists a point $r_{n}^{\prime \prime} \in\left[r_{n}^{\prime},(\delta+1) r_{n}^{\prime}\right]-E_{2}$. From

we have

$$
\frac{\log \log M\left(r_{n}^{\prime \prime}, g\right)}{\log r_{n}^{\prime \prime}} \geqq \frac{\log \log M\left(r_{n}^{\prime}, g\right)}{\log \left[(\delta+1) r_{n}^{\prime}\right]}=\frac{\log \log M\left(r_{n}^{\prime}, g\right)}{\log r_{n}^{\prime}+\log (\delta+1)^{-}},
$$

$$
\begin{aligned}
\varlimsup_{r_{n}^{\prime} \rightarrow \infty}=\frac{\log \log M\left(r_{n}^{\prime}, g\right)}{\log r_{n}^{\prime}} & =\varlimsup_{r_{n \rightarrow \infty}^{\prime}} \frac{\log \log M\left(r_{n}^{\prime}, g\right)}{\log r_{n}^{\prime}+\log (\delta+1)} \\
& \leqq \varlimsup_{r_{n \rightarrow \infty}^{\prime \prime}} \frac{\log \log M\left(r_{n}^{\prime \prime}, g\right)}{\log r_{n}^{\prime \prime}}
\end{aligned}
$$




$$
\leqq \varlimsup_{\substack{r \rightarrow \infty \\ r \in(1,+\infty)-E_{2}}} \frac{\log \log M(r, g)}{\log r} .
$$

Since $\left\{r_{n}^{\prime}\right\}$ is arbitrary, we have $\alpha \leqq \alpha_{1}$. This is a contradiction, hence $\operatorname{lm} E_{2}=\infty$.

By $\sigma(g)=\alpha<\infty$ and Theorem $\mathrm{D}$, we have

$$
\lim _{r \rightarrow \infty} \frac{\log M(r, g)}{\log \mu(r)}=1,
$$

where $\mu(r)$ is the maximum term of the power series of $g(z), \mu(r)=\left|a_{\nu_{g}(r)}\right| r^{\nu} g(r)$ By (2.4), for sufficiently large $r$,

From

$$
\log M(r, g) \leqq 2 \log \mu(r) \leqq 2 \log ^{+}\left|a_{\nu_{g}}\right|+2 \nu_{g}(r) \cdot \log r .
$$

$$
\frac{\log \log M(r, g)}{\log r} \leqq \frac{\log \nu_{g}(r)}{\log r}+\frac{\log ^{+} \log ^{+}\left|a_{\nu_{g}}\right|+2 \log 2+\log \log r}{\log r},
$$

we have

i.e.

$$
\begin{aligned}
\alpha & =\lim _{\substack{r \rightarrow \infty \\
r \in E_{2}}} \frac{\log \log M(r, g)}{\log r}=\lim _{\substack{r \rightarrow \infty \\
r \in E_{2}}} \frac{\log \log M(r, g)}{\log r} \\
& \leqq \lim _{\substack{r \rightarrow \infty \\
r \in E_{2}}} \frac{\log \nu_{g}(r)}{\log r} \leqq \varlimsup_{\substack{r \rightarrow \infty \\
r \in(0, \infty)}} \frac{\log \nu_{g}(r)}{\log r}=\alpha,
\end{aligned}
$$

$$
\lim _{\substack{r \rightarrow \infty \\ r \in E_{2}}} \frac{\log \nu_{g}(r)}{\log r}=\alpha .
$$

LEMMA 3. Suppose that $g(z)$ is an entire function with $\sigma(g)=\infty$, then there is a set $E_{2} \subset(1, \infty)$ that has infinite logarithmic measure such that

$$
\lim _{\substack{r \rightarrow \infty \\ r \in E_{2}}} \frac{\log \nu_{g}(r)}{\log r}=\infty .
$$

Proof. Using the same proof as in the upper half part of Lemma 2, we can prove Lemma 3.

LEMMA 4 (see [4]). Suppose that $u(z)$ is a meromorphic function with $\sigma(u)$ $=\beta<\infty, \varepsilon>0$ is a given constant. Then there exists a set $E_{3} \subset(1, \infty)$ that has finite logarithmic measure, such that

$$
\left|\frac{u^{(\jmath)}(z)}{u(z)}\right| \leqq r^{\jmath(\beta-1+\varepsilon)} \quad(\jmath=1, \cdots, k)
$$

hold for all $z$ satısfying $|z|=r \notin[0,1] \cup E_{3}$.

LEMMA 5. Suppose that $u(z)$ is a meromorphic function with $\sigma(u)=\beta<\infty$, ( $m$ is integer), $\varepsilon>0$ is a given constant. Then there exists a set $E_{3} \subset(1, \infty)$ that has finite logarithmic measure, such that for all $z$ satisfying $|z|=r \notin[0,1] \cup E_{3}$, 
we have

$$
\left|u(z) \cdot\left(u^{-1}(z)\right)^{(m)}\right| \leqq r^{m(\beta-1+\varepsilon)} .
$$

Proof. First we use the mathematical induction to prove

$$
u\left(\frac{1}{u}\right)^{(m)}=\sum_{\left(\rho_{1} \cdots j_{m}\right)} \alpha_{\left(j_{1} \cdots \jmath_{m}\right)}\left(\frac{u^{\prime}}{u}\right)^{\jmath_{1}} \cdots\left(\frac{u^{(m)}}{u}\right)^{\jmath_{m}},
$$

where $\alpha_{\left(j_{1} \cdots j_{m}\right)}$ is a constant, $j_{1}, \cdots, j_{m}$ satisfy $1 \cdot j_{1}+2 \cdot j_{2}+\cdots+m \cdot j_{m}=m$. For $m=1$, (2.8) holds obviously. For $m$, assume that (2.8) holds. So, we have for $m+1$,

$$
\begin{aligned}
& \left(\frac{1}{u}\right)^{(m+1)}=\left[\left(\frac{1}{u}\right)^{(m)}\right]^{\prime}=\left[\frac{1}{u} \sum_{\left(\jmath_{1} \cdots \jmath_{m}\right)} \alpha_{\left(\rho_{1} \cdots \jmath_{m}\right)}\left(\frac{u^{\prime}}{u}\right)^{\jmath_{1}} \cdots\left(\frac{u^{(m)}}{u}\right)^{\jmath_{m}}\right]^{\prime} \\
& =-\frac{u^{\prime}}{u^{2}} \sum_{\left(\rho_{1} \cdots j_{m}\right)} \alpha_{\left(j_{1} \cdots j_{m}\right)}\left(\frac{u^{\prime}}{u}\right)^{\jmath_{1}} \cdots\left(\frac{u^{(m)}}{u}\right)^{\jmath_{m}}+\frac{1}{u} \sum_{\left(\rho_{1} \cdots j_{m}\right)} \alpha_{\left(\rho_{1} \cdots \jmath_{m}\right)} \\
& \cdot\left\{\sum _ { d = 1 } ^ { m } ( \frac { u ^ { \prime } } { u } ) ^ { \jmath _ { 1 } } \cdots ( \frac { u ^ { ( d - 1 ) } } { u } ) ^ { \jmath ^ { d - 1 } } \left[j_{d}\left(\frac{u^{(d)}}{u}\right)^{\jmath_{d}-1}\left(\frac{u^{(d+1)}}{u}\right)\right.\right. \\
& \left.\left.-j_{d}\left(\frac{u^{(d)}}{u}\right)^{\jmath} d\left(\frac{u^{\prime}}{u}\right)\right] \cdot\left(\frac{u^{(d+1)}}{u}\right)^{\jmath} d+1 \cdots\left(\frac{u^{(m)}}{u}\right)^{\jmath m}\right\} \\
& =\frac{1}{u} \sum_{\left(\rho_{1} \cdots j_{m}\right)} \alpha_{\left(\jmath_{1} \cdots \jmath_{m}\right)}\left(\frac{u^{\prime}}{u}\right)^{\jmath_{1}+1} \cdots\left(\frac{u^{(m)}}{u}\right)^{\jmath_{m}}+\frac{1}{u} \sum_{\left(\rho_{1} \cdots j_{m}\right)} \alpha_{\left(j_{1} \cdots j_{m}\right)} \\
& \cdot\left\{\sum _ { d = 1 } ^ { m } \left[-j_{d}\left(\frac{u^{\prime}}{u}\right)^{\jmath_{1}+1}\left(\frac{u^{\prime \prime}}{u}\right)^{\jmath_{2}} \cdots\left(\frac{u^{(m)}}{u}\right)^{\jmath_{m}}\right.\right. \\
& \left.\left.+j_{d}\left(\frac{u^{\prime}}{u}\right)^{\jmath_{1}} \cdots\left(\frac{u^{(d)}}{u}\right)^{\jmath^{-1}}\left(\frac{u^{(d+1)}}{u}\right)^{\jmath^{d+1}+1} \cdots\left(\frac{u^{(m)}}{u}\right)^{\jmath_{m}}\right]\right\},
\end{aligned}
$$

where the indexs satisfy $1 \cdot\left(j_{1}+1\right)+2 \cdot j_{2}+\cdots+m \cdot \jmath_{m}=m+1$, or $1 \cdot j_{1}+\cdots+$ $d \cdot\left(j_{d}-1\right)+(d+1) \cdot\left(j_{d+1}+1\right)+\cdots+m \cdot j_{m}=m+1$. Therefore (2.8) holds.

Now by (2.8) and Lemma 4 , it is easy to see that Lemma 5 holds.

LEMMA 6. Suppose that $b_{0}, \cdots, b_{k-1}, H \neq 0$ are meromorphic functions, $\sigma(H)$ $=\beta<\infty$, that there are a set $E_{3} \subset(1,+\infty)$ that has finite logarithmic measure and a constant number $C_{1}>0$, such that for $|z|=r \notin[0,1] \cup E_{3}$,

$$
\left|b_{j}(z)\right| \leqq r^{C_{1}} \quad(j=0, \cdots, k-1)
$$

hold. If an entire function $g(z)$ solves the equation

$$
g^{(k)}+b_{k-1} g^{(k-1)}+\cdots+b_{0} g=H,
$$

then $\sigma(g)<\infty$.

Proof. Assume that $\sigma(g)=\infty, \mu(r)$ denotes the maximum term of the power series of $g(z), \nu_{g}(r)$ denotes the centralindex of $g(z)$. By Lemma 3, we know that there is a set $E_{2} \subset(1, \infty)$ that has infinite logarithmic measure such that 


$$
\lim _{\substack{r \rightarrow \infty \\ r \in E_{2}}} \frac{\log \nu_{g}(r / 2)}{\log (r / 2)}=\infty
$$

Since $\nu_{g}(r)$ is a step function in $r$, we can assume that $t_{g}\left(j=0,1, \cdots, 0=t_{0}<t_{1}\right.$ $\left.<t_{2}<\cdots\right)$ are discontinuous points of $\nu_{g}(r)$. As $t \in\left(t_{\jmath}, t_{j+1}\right)$, we have $\mu(t)=\left|a_{\nu_{g}}(t)\right|$ $\cdot t^{\nu} g(t)$, where centralindex $\nu_{g}(t)=m$ is fixed constant. Hence

$$
\mu^{\prime}(t)=m\left|a_{m}\right| t^{m-1}=\mu \cdot \nu_{g}(t) / t
$$

holds for $t \in\left(t_{j}, t_{j+1}\right)$. Since $\mu(t)$ is a continuous function, we have for $r>2$

$$
\begin{aligned}
\log \mu(r)-\log \mu(1) & =\int_{1}^{r}\left[\mu^{\prime}(t) / \mu(t)\right] \mathrm{d} t \\
& =\int_{1}^{r}\left[\nu_{g}(t) / t\right] \mathrm{d} t>\int_{r / 2}^{r}\left(\nu_{g}(t) / t\right) \mathrm{d} t \geqq \nu_{g}(r / 2) \cdot \log 2 .
\end{aligned}
$$

By Cauchy's inequality, it is easy to know that $\mu(r) \leqq M(r, g)$. So,

$$
\nu_{g}(r / 2) \cdot \log 2 \leqq \log M(r, g)-\log \mu(1) .
$$

For a given large $\alpha$ such that

$$
\alpha>\max \left\{C_{1}, \beta\right\}+k,
$$

by (2.11), (2.12), we obtain

$$
\begin{aligned}
& \nu_{g}(r) \geqq \nu_{g}(r / 2) \geqq(r / 2)^{\alpha}=C_{2} r^{\alpha}, \\
& M(r, g) \geqq C_{3} \cdot \exp \left\{C_{1} r^{\alpha}\right\}
\end{aligned}
$$

for $r \in E_{2}, r \rightarrow \infty$, where $C_{2}, C_{3}, C_{4}$ are positive constants.

From the Wiman-Valiron theory (see [6], [8], [9]) we have basic formulas

$$
\frac{g^{(j)}(z)}{g(z)}=\left(\frac{\nu_{g}(r)}{z}\right)^{\jmath}(1+o(1)) \quad(j=1, \cdots, k)
$$

where $|z|=r,|g(z)|=M(r, g), r \notin E_{4}, \int_{E_{4}} \frac{\mathrm{d} r}{r}<\infty$.

By Lemma 1, we have

$$
|H(z)| \leqq \exp \left\{r^{\beta+1 / 2}\right\}
$$

for $|z|=r \in[1,+\infty)-E_{1}, \int_{E_{1}} \frac{\mathrm{d} r}{r}<\infty$.

Now, we take sufficiently large $|z|=r \in E_{2}-\left(E_{1} \cup E_{3} \cup E_{4}\right),|g(z)|=M(r, g)$, logarithmic measure $\operatorname{lm}\left[E_{2}-\left(E_{1} \cup E_{3} \cup E_{4}\right)\right]=\infty$. (2.10) and (2.16) give

$$
\left(\frac{\nu_{g}(r)}{z}\right)^{k}(1+o(1))+b_{k-1}\left(\frac{\nu_{g}(r)}{z}\right)^{k-1}(1+o(1))+\cdots+b_{0}=\frac{H(z)}{g(z)},
$$




$$
\begin{aligned}
\frac{\nu_{g}(r)}{z^{k}}(1+o(1))= & \frac{H(z)}{g(z) \nu_{g}^{k-1}(r)}-\frac{b_{k-1}}{z^{k-1}}(1+o(1)) \\
& -\frac{b_{k-2}}{z^{k-2} \nu_{g}(r)}(1+o(1))-\cdots-\frac{b_{0}}{\boldsymbol{\nu}_{g}^{k-1}(r)}
\end{aligned}
$$

By (2.13)-(2.15), (2.17), we have

$$
\begin{aligned}
& \frac{|H(z)|}{|g(z)|}=\frac{|H(z)|}{M(r, g)} \leqq \frac{1}{C_{3}} \exp \left\{r^{\beta+(1 / 2)}-C_{4} r^{\alpha}\right\} \rightarrow 0, \\
& \left|\frac{b_{j}(z)}{z^{j} \nu_{g}^{k-1-j}(r)}\right| \rightarrow 0 \quad(j=0, \cdots, k-2)
\end{aligned}
$$

hold for $|z|=r \in E_{2}-\left(E_{1} \cup E_{3} \cup E_{4}\right), r \rightarrow \infty$. And (2.14), (2.19) and (2.20) give

$$
\begin{aligned}
& \left|\frac{H(z)}{g(z) \cdot \nu_{g}^{k-1}(r)}-\frac{b_{k-1}}{z^{k-1}}(1+o(1))-\frac{b_{k-2}}{z^{k-2} \nu_{g}(r)}(1+o(1))-\cdots-\frac{b_{0}}{\nu_{g}^{k-1}(r)}\right| \\
= & O\left(\frac{b_{k-1}}{z^{k-1}}\right)=O\left(r^{C_{1}-k+1}\right) .
\end{aligned}
$$

On the other hand, by (2.14), we have

$$
\left|\frac{\nu_{g}(r)}{z^{k}}(1+o(1))\right| \geqq C_{2} r^{\alpha-k}>r^{C_{1}}
$$

for $r \in E_{2}, r \rightarrow \infty$. And (2.21) contradicts (2.22) by (2.18). Therefore $\sigma(g)<\infty$.

LEMMA 7. Suppose that $b_{k_{-}}(j=1, \cdots, k)$ are rational functions having $a$ pole at $\infty$ of order $n_{k-\jmath} \geqq 0, k \geqq 0, H(z) \neq 0$ is a meromorphic function with $\sigma(H)$ $=\beta$. If (1.1) has a meromorphic solution $f$, then

(a) If $1+\max _{1 \leq j \leq k} n_{k-j} / j<\beta<\infty$, then $\sigma(f)=\beta$.

(b) If $\beta \leqq 1+\max _{1 \leqq \jmath \leqq k} n_{k-j} / j$, then $\beta \leqq \sigma(f) \leqq 1+\max _{1 \leqq j \leq k} n_{k-j} / j$.

Proof. We have $\sigma(f) \geqq \beta$ from (1.1). By (1.1) and fact that $b_{k-\jmath}(j=1, \cdots, k)$ have only finitely many poles, we know that if $|z|(<\infty)$ is sufficiently large, then either $f$ and $H$ are both analytic at $z$, or $f$ has a pole at $z$ of order $m_{1}$ if and only if $H$ has a pole at $z$ of order $m_{1}+k$. So,

$$
\bar{\lambda}(1 / f)=\bar{\lambda}(1 / H) \text {. }
$$

From

$$
n(r, f) \leqq n(r, H)+O(1) \text { and } n(r, H) \leqq(k+1) n(r, f)+O(1),
$$

it follows that

$$
\lambda(1 / f)=\lambda(1 / H) .
$$

Set $f(z)=g(z) /\left(z^{m_{2}} \cdot u(z)\right)=g(z) / u_{1}(z)$, where $m_{2}$ is a nonnegative integer, $g(z)$ is an entire function, $u(z)$ is a canonical product (or polynomial) formed with 
the nonzero poles $\left\{z_{\jmath}: j=1,2, \cdots\right\} \quad\left(\left|z_{j}\right|=r_{\jmath}, 0<r_{1} \leqq r_{2} \leqq \cdots\right)$ of $f, u_{1}(z)=z^{m_{2}} u(z)$, then $\lambda\left(u_{1}\right)=\sigma\left(u_{1}\right)=\lambda(1 / f)=\lambda(1 / H) \leqq \beta$.

Now we suppose that $\sigma(f)=\alpha>\beta$. By $f=g / u_{1}$ and $\sigma\left(u_{1}\right) \leqq \beta$, we have $\sigma(g)=\sigma(f)=\alpha$. For any given $\varepsilon(0<2 \varepsilon<\alpha-\beta)$, by Lemma 1, it follows that there is a set $E_{1} \subset(1,+\infty)$ that has finite logarithmic measure, such that

$$
\left|1 / u_{1}(z)\right| \leqq \exp \left\{r^{\beta+\varepsilon}\right\}
$$

holds for $|z|=r \notin[0,1] \cup E_{1}, r \rightarrow \infty$. From (2.24), (2.25) and the fact that the poles of $f$ can only occur at poles of $H$ except at most finitely many poles, it follows that

$$
|H(z)| \leqq \exp \left\{r^{\beta+\varepsilon}\right\}
$$

holds for $|z|=r \notin[0,1] \cup E_{1}, r \rightarrow \infty$. By $f(z)=g(z) / u_{1}(z)$, we have for $n=1, \cdots, k$

$$
\frac{f^{(n)}}{f}=\frac{g^{(n)}}{g}+C_{n}^{1} \cdot u_{1}\left(\frac{1}{u_{1}}\right)^{\prime} \frac{g^{(n-1)}}{g}+\cdots+C_{n}^{(n-1)} \cdot u_{1}\left(\frac{1}{u_{1}}\right)^{(n-1)} \frac{g^{\prime}}{g}+u_{1}\left(\frac{1}{u_{1}}\right)^{(n)}
$$

where $C_{n}^{j}(j=1, \cdots, n)$ are the usual notation for the binomial coefficients. (2.27) and (1.1) give

$$
\frac{g^{(k)}}{g}+d_{k-1} \frac{g^{(k-1)}}{g}+\cdots+d_{1} \frac{g^{\prime}}{g}+d_{0}=\frac{H \cdot u_{1}}{g}
$$

where

$$
\begin{array}{r}
d_{k-\jmath}=C_{k}^{\jmath} \cdot u_{1}\left(\frac{1}{u_{1}}\right)^{(j)}+b_{k-1} \cdot C_{k-1}^{\jmath-1} u_{1}\left(\frac{1}{u_{1}}\right)^{(\jmath-1)}+\cdots+b_{k-\jmath+1} \cdot C_{k-\jmath+1}^{1} u_{1}\left(\frac{1}{u_{1}}\right)^{\prime}+b_{k-\jmath} \\
(j=1, \cdots, k)
\end{array}
$$

By $\sigma\left(u_{1}\right) \leqq \beta$ and Lemma 6 , there is a set $E_{3} \subset(1, \infty)$ that has finite logarithmic measure, such that for $|z|=r \notin[0,1] \cup E_{3}$, for $j=1, \cdots, k$, we have

$$
\left|u_{1}(z)\left(u_{1}^{-1}(z)\right)^{(\jmath)}\right| \leqq r^{\jmath(\beta-1+\varepsilon)} .
$$

(a) Suppose $1+\max _{1 \leq j \leq k} n_{k-j} / j<\beta<\infty$. Now we prove $\sigma(f)=\alpha>\beta$ fails. From (2.30) and

$$
n_{k-\jmath} \leqq j(\beta-1) \quad(\jmath=1, \cdots, k),
$$

we have for $|z|=r \notin[0,1] \cup E_{3}, r \rightarrow \infty$.

$$
\left|b_{k-q}(z) u_{1}(z)\left(u_{1}^{-1}(z)\right)^{\jmath-q}\right| \leqq r^{n_{k-q^{+}(\jmath-q)(\beta-1+\varepsilon)}<r^{\jmath(\beta-1+\varepsilon)}} \quad(q=1, \cdots, j) .
$$

(2.29) and (2.32) give for $|z|=r \notin[0,1] \cup E_{3}, r \rightarrow \infty$

$$
\left|d_{k-j}(z)\right|=O\left(r^{j(\beta-1+\varepsilon)}\right) \quad(j=1, \cdots, k) .
$$

By Lemma 6 and (2.28), (2.33), we have $\sigma(g)=\alpha<\infty$. From Lemma 2 and $\sigma(g)<\infty$, there is a set $E_{2} \subset(1, \infty)$ that has infinite logarithmic measure such that 


$$
\lim _{\substack{r \rightarrow \infty \\ r \in E_{2}}} \frac{\log \log M(r, g)}{\log r}=\lim _{\substack{r \rightarrow \infty \\ r \in E_{2}}} \frac{\log \nu_{g}(r)}{\log r}=\alpha .
$$

From the Wiman-Valiron theory, there is a set $E_{4} \subset(1, \infty)$ that has finite logarithmic measure, such that for $|z|=r \notin E_{4},|g(z)|=M(r, g)$, (2.16) holds. By (2.34), we have

$$
M(r, g) \geqq \exp \left\{r^{\alpha-\varepsilon}\right\}
$$

for $|z|=r \in E_{2}-\left(E_{1} \cup E_{3} \cup E_{4} \cup[0,1]\right)$ and sufficiently large $r$. From (2.26), (2.35) and $\left|u_{1}(z)\right| \leqq \exp \left\{r^{\beta+\varepsilon}\right\}(|z|=r \rightarrow \infty)$, and $\beta+\varepsilon<\alpha-\varepsilon$, we get

$$
\left|\frac{u_{1}(z) \cdot H(z)}{g(z)}\right|=\left|\frac{u_{1}(z) \cdot H(z)}{M(r, g)}\right| \leqq \exp \left\{2 r^{\beta+\varepsilon}-r^{\alpha-\varepsilon}\right\} \rightarrow 0
$$

for $|z|=r \in E_{2}-\left(E_{1} \cup E_{3} \cup E_{4} \cup[0,1]\right),|g(z)|=M(r, g), r \rightarrow \infty$. By (2.34),

$$
\nu_{g}(r)=r^{\alpha+o(1)}
$$

holds for $|z|=r \in E_{2}-\left(E_{1} \cup E_{3} \cup E_{4} \cup[0,1]\right), r \rightarrow \infty$. Since the logarithmic measure of $E_{2}-\left(E_{1} \cup E_{3} \cup E_{4} \cup[0,1]\right), \operatorname{lm}\left[E_{2}-\left(E_{1} \cup E_{3} \cup E_{4} \cup[0,1]\right)\right]=\infty$, and by (2.16), (2.28), (2.33), (2.36), we obtain

$$
\begin{gathered}
\left(\frac{\nu_{g}(r)}{z}\right)^{k}(1+o(1))+O\left(r^{\beta-1+s}\right)\left(\frac{\nu_{g}(r)}{z}\right)^{k-1}(1+o(1))+\cdots+ \\
O\left(r^{(k-1)(\beta-1+\varepsilon)}\right) \frac{\nu_{g}(r)}{z}(1+o(1))+O\left(r^{k(\beta-1+\varepsilon)}\right)=o(1)
\end{gathered}
$$

for $|z|=r \in E_{2}-\left(E_{1} \cup E_{3} \cup E_{4} \cup[0,1]\right),|g(z)|=M(r, g), r \rightarrow \infty$. By (2.37), (2.38) and $0<2 \varepsilon<\alpha-\beta$, for $|z|=r \in E_{2}-\left(E_{1} \cup E_{3} \cup E_{4} \cup[0,1]\right),|g(z)|=M(r, g), r \rightarrow \infty$, it is easy to see that there is only one term $\left(\nu_{g}(r) / z\right)^{k}(1+o(1))$ with the degree $k(\alpha-1)$ being the highest one among all terms of (2.38). This is impossible. Therefore, $\sigma(f)=\beta$.

(b) Suppose that $\beta \leqq 1+\max _{1 \leqq j \leq k} n_{k-j} / j$. Now we prove $1+\max _{1 \leqq \jmath \leqq k} n_{k-j} / j$ $<\sigma(f)=\alpha$ fails. We set $1+\max _{1 \leq j \leq k} n_{k-j} / \jmath=m<\alpha$, then

$$
n_{k-\jmath} \leqq j(m-1) \quad(\jmath=1, \cdots, k) \text { and } \beta \leqq m .
$$

By $\beta \leqq m$ and (2.30), (2.39) we have for $|z|=r \notin E_{3} \cup[0,1], r \rightarrow \infty$

$$
\begin{aligned}
\left|b_{k-q}(z) u_{1}(z)\left(u_{1}^{-1}(z)\right)^{(\jmath-q)}\right| & \leqq r^{n_{k-q^{+}(\jmath-q)(\beta-1+\varepsilon)}} \\
& \leqq r^{q(m-1)+(\jmath-q)(\beta-1+\varepsilon)} \\
& <r^{\jmath(m-1+\varepsilon)} \quad(q=1, \cdots, j) .
\end{aligned}
$$

(2.29) and (2.40) give for $|z|=r \notin E_{3} \cup[0,1], r \rightarrow \infty$

$$
\left|d_{k-j}(z)\right|=O\left(r^{\jmath(m-1+\varepsilon)}\right) \quad(j=1, \cdots, k) .
$$


By Lemma 6 and (2.28), (2.41), we have $\sigma(f)=\alpha<\infty$. Using the same reasoning as in (a), it is easy to know that (2.34)-(2.37) hold. Since $\operatorname{lm}\left[E_{2}-\left(E_{1} \cup E_{3} \cup E_{4}\right.\right.$ $\cup[0,1])]=\infty$ and (2.16), (2.28), (2.36), (2.41), we have

$$
\begin{gathered}
\left(\frac{\nu_{g}(r)}{z}\right)^{k}(1+o(1))+O\left(r^{m-1+\varepsilon}\right)\left(\frac{\nu_{g}(r)}{z}\right)^{k-1}(1+o(1))+\cdots+ \\
O\left(r^{(k-1)(m-1+\varepsilon)} \frac{\nu_{g}(r)}{z}(1+o(1))+O\left(r^{k(m-1+\varepsilon)}\right)=o(1)\right.
\end{gathered}
$$

for $|z|=r \in E_{2}-\left(E_{1} \cup E_{3} \cup E_{4} \cup[0,1]\right),|g(z)|=M(r, g), r \rightarrow \infty$. From (2.37), (2.42) and $\alpha>m$, for $|z|=r \in E_{2}-\left(E_{1} \cup E_{3} \cup E_{4} \cup[0,1]\right),|g(z)|=M(r, g), r \rightarrow \infty$, it is easy to see that there is only one term $\left(\nu_{g}(r) / z\right)^{k}(1+o(1))$ with the degree $k(\alpha-1)$ being the highest one among all terms of (2.42). This is impossible. Therefore, $\beta \leqq \sigma(f) \leqq m$.

LEMMA 8. Suppose that $\beta$ is a positive integer and $\beta>1, B_{k-\jmath}(\jmath=1, \cdots, k)$ are rational functions having a pole at $\infty$ of order $n_{k-\jmath}=j(\beta-1), U \not \equiv 0$ is a meromorphic function with $\sigma(U)<\beta$. If the equation

$$
y^{(k)}+B_{k-1} y^{(k-1)}+\cdots+B_{0} y=U
$$

has a meromorphic solution $y$, then $\sigma(y)=\beta$ except at most one possible exceptional meromorphic solution $y_{0}$ with $\sigma\left(y_{0}\right)<\beta$.

If $y \not \equiv 0$ is a meromorphic solution of the equation

$$
y^{(k)}+B_{k-1} y^{(k-1)}+\cdots+B_{0} y=0
$$

that is the corresponding homogeneous differential equation of (2.43), the $\sigma(y)=\beta$.

Proof. Set $\sigma(y)=\alpha$, then $\alpha \geqq \sigma(U)=d$ by (2.43). Now assume that $\sigma(y)=$ $\alpha>d$. Set $y(z)=g(z) / u_{1}(z)$ where $g(z), u_{1}(z)$ are functions defined in the same way as in Lemma 7 . Using the same reasoning as in Lemma 7 , we have $\sigma\left(u_{1}\right) \leqq d$ and

$$
\left|u_{1}(z)\right| \leqq \exp \left\{r^{d+\varepsilon}\right\} \quad(|z|=r \rightarrow \infty) .
$$

And there is a set $E_{4} \subset(1, \infty)$ has finite logarithmic measure such that (2.16) holds for $|z|=r \notin[0,1] \cup E_{4}, \quad|g(z)|=M(r, g)$. For any given $\varepsilon(0<2 \varepsilon<$ $\min \{\alpha-d, \beta-d\})$, there is a set $E_{1} \subset(1,+\infty)$ that has finite logarithmic measure such that for $|z|=r \notin E_{1} \cup[0,1], r \rightarrow \infty$

$$
|U(z)| \leqq \exp \left\{r^{d+\varepsilon}\right\} .
$$

By Lemma 5 and the hypotheses, there is a set $E_{3} \subset(1,+\infty)$ that has finite logarithmic measure such that for $|z|=r \notin[0,1] \cup E_{3}$,

$$
\left|u_{1}(z)\left(u_{1}^{-1}(z)\right)^{(\jmath)}\right| \leqq r^{\jmath(d-1+\varepsilon)} \quad(j=1, \cdots, k) .
$$


Substituting $y=g / u_{1}$ into (2.43), we get

$$
\frac{g^{(k)}}{g}+D_{k-1} \frac{g^{(k-1)}}{g}+\cdots+D_{0}=\frac{U \cdot u_{1}}{g}
$$

where

$$
\begin{array}{r}
D_{k-\jmath}=C_{k}^{\jmath} u_{1}\left(u_{1}^{-1}\right)^{(\jmath)}+B_{k-1} C_{k-1}^{\jmath-1} u_{1}\left(u_{1}^{-1}\right)^{(\jmath-1)}+\cdots+B_{k-\jmath+1} C_{k-\jmath+1}^{1} u_{1}\left(u_{1}^{-1}\right)^{\prime}+B_{k-\jmath} \\
(j=1, \cdots, k) .
\end{array}
$$

Since (2.47), (2.49) and $B_{k-j}(z)=C_{k-j} \cdot z^{\jmath(\beta-1)}(1+o(1))\left(\left(C_{k-\jmath} \neq 0\right.\right.$ is constant $)(\jmath=$ $1, \cdots, k)$, and $\beta>d+\varepsilon$, we have

$$
D_{k-\jmath}=C_{k-j} z^{\jmath(\beta-1)}(1+o(1)) \quad(j=1, \cdots, k) .
$$

for $|z|=r \notin E_{3} \cup[0,1], r \rightarrow \infty$.

By (2.48), (2.50) and Lemma 6, we know that $\sigma(g)=\alpha<\infty$. By Lemma 2, there is a set $E_{2} \subset(1,+\infty)$ that has infinite logarithmic measure, such that (2.34) holds. Using the same reasoning as in Lemma 7, we have

$$
\left|\frac{u_{1}(z) \cdot U(z)}{g(z)}\right|=\left|\frac{u_{1}(z) \cdot U(z)}{M(r, g)}\right| \leqq \exp \left\{2 r^{d+\varepsilon}-r^{\alpha-\varepsilon}\right\} \rightarrow 0 .
$$

for $|z|=r \in E_{2}-\left([0,1] \cup E_{1} \cup E_{3} \cup E_{4}\right),|g(z)|=M(r, g), r \rightarrow \infty$. By (2.48), (2.50), (2.51), and (2.16), we get

$$
\begin{gathered}
\left(\frac{\nu_{g}(r)}{z}\right)^{k}(1+o(1))+C_{k-1} z^{\beta-1}\left(\frac{\nu_{g}(r)}{z}\right)^{k-1}(1+o(1))+\cdots+ \\
C_{1} z^{(k-1)(\beta-1)}\left(\frac{\nu_{g}(r)}{z}\right)(1+o(1))+C_{0} z^{k(\beta-1)}(1+o(1))=o(1)
\end{gathered}
$$

for $|z|=r \in E_{2}-\left([0,1] \cup E_{1} \cup E_{3} \cup E_{4}\right), \quad|g(z)|=M(r, g), \quad r \rightarrow \infty$. By (2.34), we have

$$
\nu_{g}(r)=r^{\alpha+o(1)}
$$

for $|z|=r \in E_{2}-\left([0,1] \cup E_{1} \cup E_{3} \cup E_{4}\right),|g(z)|=M(r, g), r \rightarrow \infty$. By (2.53) and $\varepsilon$ arbitrarily small, we see that the degrees of all terms of the left of (2.52) are respectively

$$
k(\alpha-1),(k-j)(\alpha-1)+j(\beta-1) \quad(j=1, \cdots, k) .
$$

From the Wiman-Valiron theory, we get $\alpha=\beta$, i.e. $\sigma(y)=\sigma(g)=\beta$.

Using the same manner as above, we can prove that if $y(z) \neq 0$ is a meromorphic solution of $(2.44)$, then $\sigma(y)=\beta$.

If $y_{0}$ and $y_{1}\left(y_{1} \not \equiv y_{0}\right)$ are both meromorphic solutions of (2.43) with $\sigma\left(y_{j}\right)<\beta$ $(j=0,1)$, then $\sigma\left(y_{1}-y_{0}\right)<\beta$. But $y_{1}-y_{0} \neq 0$ is a meromorphic solution of (2.44), we have $\sigma\left(y_{1}-y_{0}\right)=\beta$ by the proof given above. Therefore, (2.43) has at most one exceptional meromorphic solution $y_{0}$ with $\sigma\left(y_{0}\right)<\beta$. 
THE ZERO, POLE AND ORDER OF MEROMORPHIC SOLUTIONS

LEMMA 9. Suppose that $b_{k-\jmath}(\jmath=1, \cdots, k)$ are rational functions having a pole at $\infty$ of order $n_{k-\jmath} \geqq 0, k \geqq 1, H(z)$ is a meromorphic function, $\sigma(H)=\beta<\infty$. If $f$ is a meromorphic solution of (1.1), then

$$
\max \{\lambda(f), \lambda(1 / f)\} \geqq \max \{\lambda(H), \lambda(1 / H)\} .
$$

Proof. Set $f=g / u_{1}$, where $g$ and $u_{1}$ are functions defined in the same way as in Lemma 7. Using the same method as in the proof of Lemma 7, we know that (2.28), (2.33) hold. By Lemma 6, we have $\sigma(g)<\infty$, hence $\sigma(f)<\infty$.

Using the same reasoning as in Lemma 7 of [3] we can prove (2.54) holds.

LEMMA 10 (see [3]). Suppose that $b_{k_{-}}(\jmath=1, \cdots, k)$ are rational functions, $H \not \equiv$ is a meromorphic function with $\sigma(H)<\infty$. If $f$ is a meromorphic solution of (1.1) such that $\sigma(H)<\sigma(f)<\infty$, then $\bar{\lambda}(f)=\lambda(f)=\sigma(f)$.

\section{Proof of Theorem 1}

(a) By Lemma 7, we have $\sigma(f)=\beta$.

(b) By (2.23) and (2.24) in proof of Lemma 7, we have $\bar{\lambda}(1 / f)=\bar{\lambda}(1 / H)$, $\lambda(1 / f)=\lambda(1 / H)$. If $\lambda(H)>\lambda(1 / H)$, we have $\lambda(f) \geqq \lambda(H)$ by Lemma 9 .

(c) If $\beta>\max \{\lambda(H), \lambda(1 / H)\}$, then set $H=U e^{p}$, where $U=z^{s}\left(v_{1} / v_{2}\right)$ (s is an integer), $v_{1}$ and $v_{2}$ are canonical products (or polynomials) formed respectively with the nonzero zeros and nonzero poles of $H, \sigma(U)=\max \{\lambda(H), \lambda(1 / H)\}<\beta$, $p$ is a polynomial with $\operatorname{deg} p=\beta$.

Now set $f=g e^{p}$, then $f(z)$ and $g(z)$ have the same zeros and poles. From

$$
f^{(m)}=\left\{g^{(m)}+m p^{\prime} g^{(m-1)}+\sum_{\jmath=2}^{m} C_{m}^{\jmath}\left(p^{\prime}\right)^{\jmath}+H_{\jmath-1}\left(p^{\prime}\right) g^{(m-\jmath)}\right\} e^{p}
$$

where $m=2,3, \cdots, k, H_{\jmath_{-1}}\left(p^{\prime}\right)$ are differential polynomials in $p^{\prime}$ and its derivatives of total degree $j-1$ with constant coefficients. It is easy to see that the derivatives of $H_{\jmath-1}\left(p^{\prime}\right)$ as to $z$ are of the same form $H_{\jmath-1}\left(p^{\prime}\right)$. Substituting $f=g e^{p}, H=U e^{p}$ into (1.1), we have by $(3.1)$

$$
g^{(k)}+B_{k-1} g^{(k-1)}+\cdots+B_{0} g=U
$$

where

$$
\left\{\begin{array}{rr}
B_{k-\jmath}=b_{k-\jmath}+(k-\jmath+1) b_{k-\jmath+1} p^{\prime}+\sum_{n=2}^{\jmath} b_{k-\jmath+n} C_{k-\jmath+n}^{n}\left(p^{\prime}\right)^{n}+H_{n-1}\left(p^{\prime}\right) \\
B_{k-1}=b_{k-1}+k p^{\prime} & \left(j=2, \cdots, k, b_{k} \equiv 1\right)
\end{array}\right.
$$

Since $\beta>1+\max _{1 \leqq \jmath \leq k} n_{k-j} / j$, the degree $j(\beta-1)$ of the term $b_{k} C_{k}^{\jmath}\left(p^{\prime}\right)^{\jmath}=C_{k}^{j}\left(p^{\prime}\right)^{\jmath}$ $(n=j)$ is the highest one in the first equality of (3.3). Hence $B_{k-j}(j=2, \cdots, k)$ must have a pole at $\infty$ of order $j(\beta-1) . \quad B y \operatorname{deg} p^{\prime}=\beta-1>n_{k-1}$, the rational function $B_{k-1}$ has a pole at $\infty$ of order $1 \cdot(\beta-1)$. By Lemma 8 , we see that all meromorphic solutions of (3.2) satisfy $\sigma(g)=\beta$ except at most one possible 
exceptional meromorphic solution $g_{0}$ with $\sigma\left(g_{0}\right)<\beta$. If $\sigma\left(g_{0}\right)<\beta$, then $\lambda\left(g_{0}\right)<\beta$. If $\sigma(g)=\beta$, by $\sigma(U)<\beta$ and Lemma 10 , we have $\bar{\lambda}(g)=\lambda(g)=\sigma(g)=\beta$. Therefore, all meromorphic solutions $f=g e^{p}$ of (1.1) satisfy $\bar{\lambda}(f)=\lambda(f)=\sigma(f)=\beta$ except at most one possible one $f_{0}=g_{0} e^{p}$ satisfying $\lambda\left(f_{0}\right)<\beta$.

\section{Proof of Theorem 2}

(a) By Lemma 7 , we have $\beta \leqq \sigma(f) \leqq 1+\max _{1 \leqq j \leqq k} n_{k-j} / \jmath$.

(b) Using the same reasoning as in the proof of Theorem 1 (b), we have (b).

(c) If $\sigma(f)>\sigma(H)$, then by Lemma 10, we have $\bar{\lambda}(f)=\lambda(f)=\sigma(f)$.

Acknowledgement. The author would like to thank the referees for valuable suggestions to improve my paper.

\section{REFERENCES}

[1] S. Bank And I. Laine, On the oscillation theory of $f^{\prime \prime}+A f=0$ where $A$ is entire, Trans. Amer. Math. Soc., 273 (1982), 351-361.

[2] R.P. BoAs, Entire Functions, Academic Press INC., New York, 1954.

[3] Z.-X. Chen AND S.-A. GAO, On the complex oscillation of non-homogeneous linear differential equations with meromorphic coefficients, Kodai Math. J., 15 (1992), 65-78.

[4] G. GundERSEN, Estimates for the logarithmic derivative of a meromorphic function, plus similar estimates, J. London Math. Soc. (2), 37 (1988), 88-104.

[5] W. Hayman, Meromorphic Functions, Clarendon Press, Oxford, 1964.

[6] Y.-Z. He AND X.-Z. XiaO, Algebroid Functions and Ordinary Differential Equations, Science Press, 1988 (in Chinese).

[7] R.-F. Li, C.-J. DaI AND G.-D. Song, The Continuation of Theory of Functions of a Complex Variable, Higher Education Press, 1986 (in Chinese).

[8] G. VALIRON, Lectures on the General Theory of Integral Functions, Clelsea, New York, 1949.

[9] G. Valiron, Functions Analytiques, Presses Universitaires de France, Paris, 1954.

DePaRtMENT OF MATHEMATICS

JiANGXI NORMAL UNIVERSITY

NANCHANG, 330027

JIANGXI PROV.

P. R. CHINA 